\title{
A Detailed Observation of a LMC SNR, DEM L241, with XMM-Newton
}

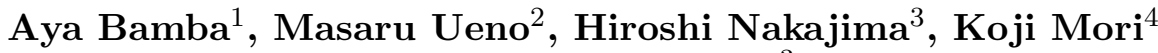 and Katsuji Koyama ${ }^{3}$}

\author{
${ }^{1}$ RIKEN (The Institute of Physical and Chemical Research) 2-1, Hirosawa, Wako, Saitama \\ 351-0198, Japan \\ email: bamba@crab.riken.jp \\ ${ }^{2}$ Department of physics, Faculty of Science, Tokyo Institute of Technology 2-12-1, \\ Oo-okayama, Meguro-ku, Tokyo 152-8551, Japan \\ email: masaru@hp.phys.titech.ac.jp \\ ${ }^{3}$ Department of Physics, Graduate School of Science, Kyoto University, Sakyo-ku, Kyoto \\ 606-8502, Japan \\ email:nakajima@cr.scphys.kyoto-u.ac.jp, koyama@cr.scphys.kyoto-u.ac.jp \\ ${ }^{4}$ Department of Applied Physics, Faculty of Engineering University of Miyazaki, 1-1 Gakuen \\ Kibana-dai Nishi Miyazaki, 889-2192, Japan \\ email: mori@astro.miyazaki-u.ac.jp
}

\begin{abstract}
We report on an XMM-Newton observation of the supernova remnant (SNR) DEM L241 in the Large Magellanic Cloud. In the soft band image, the emission shows an elongated structure, like a killifish (Head and Tail), with a central point source, named as XMMU J053559.3-673509 (Eye). The Eye's spectrum is well reproduced with a power-law model. The source has neither significant coherent pulsations nor time variabilities. Its luminosity and spectrum remind us that the source might be a pulsar and/or pulsar wind nebula in DEM L241. The spectra of Head and Tail are well reproduced by a non-equilibrium ionization plasma model with over-abundant Ne and under-abundant Fe, suggesting that the progenitor of DEM L241 is a very massive star.
\end{abstract}

Keywords. supernova remnants, X-rays: individual (DEM L241), X-rays: individual (XMMU J053559.3-673509).

\section{Introduction}

Supernovae and supernova remnants (SNRs) shape and enrich the chemical and dynamical structure of the interstellar medium and clouds. The Large Magellanic Cloud (LMC) is the best galaxy for the systematic study of SNRs, thanks to the known distance (Feast 1999). In the LMC, we pointed out DEM L241 (0536-67.6), which was identified by Mathewson et al. (1985), and observed with XMM-Newton. The total exposure is $45 \mathrm{ks}$ for MOS and $43 \mathrm{ks}$ for pn, respectively. The details are in Bamba et al. (2005).

\section{Results}

Figure 1 shows the XMM-Newton MOS $1+2$ images of DEM L241 in the (a) 0.5$2.0 \mathrm{keV}$ and (b) 2.0-9.0 keV bands. In the soft band image, we can see a diffuse structure elongated from southeast to northwest with the size of $\sim 1.5^{\prime} \times 3^{\prime}$, corresponding to $22 \mathrm{pc} \times 44 \mathrm{pc}$ at $50 \mathrm{kpc}$. The shape is like a killifish, with double peaked feature on its "Head" and "Tail". In addition to the body of the fish, there is a point source like an "Eye" of the fish. On the other hand, only Eye can be seen in the hard band image. We found no candidate of the counterpart of Eye in the SIMBAD database, and named it as XMMU J053559.3-673509. 

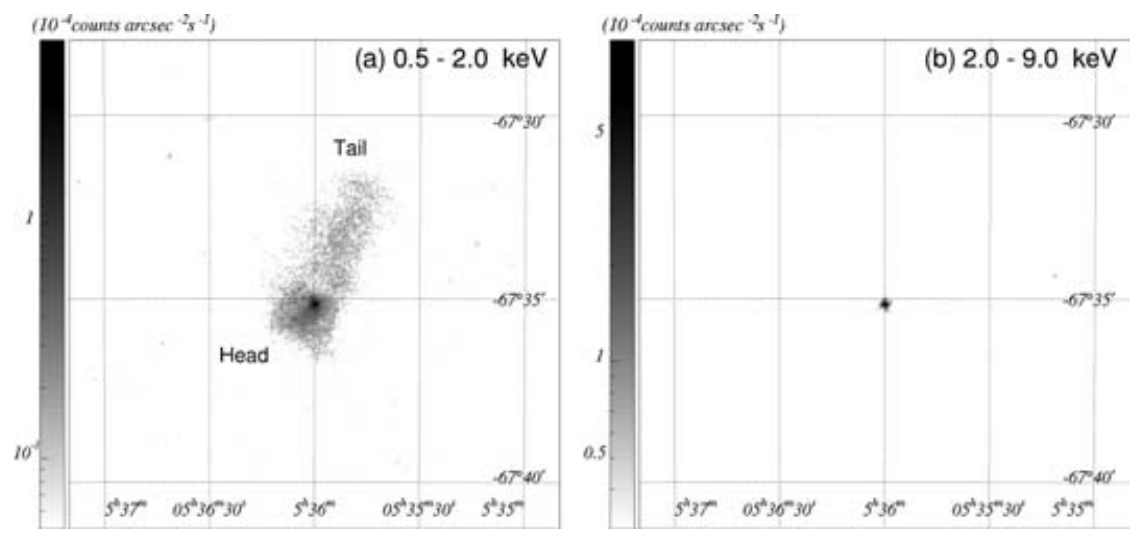

Figure 1. MOS $1+2$ images in the (a) $0.5-2.0 \mathrm{keV}$ and (b) $2.0-9.0 \mathrm{keV}$ bands.

Table 1. Best-fit parameters for the Head, Tail, and Eye

\begin{tabular}{|c|c|c|c|}
\hline Parameters & Head & Tail & Eye \\
\hline$k T_{e} / \Gamma[\mathrm{keV} /-] \ldots$ & $0.54(0.46-0.57)$ & $0.43(0.38-0.50)$ & $1.57(1.51-1.62)$ \\
\hline$n_{e} t_{p}\left[10^{11} \mathrm{~cm}^{-3} \mathrm{~s}\right]$. & $2.4(1.9-4.3)$ & $3.4(2.1-5.2)$ & - \\
\hline$[\mathrm{Ne} / \mathrm{H}] \ldots \ldots \ldots$ & $0.64(0.60-0.69)$ & $0.58(0.54-0.63)$ & - \\
\hline$[\mathrm{Si} / \mathrm{H}]$. & $(<0.05)$ & $(<0.07)$ & - \\
\hline$[\mathrm{Fe} / \mathrm{H}]$ & $0.09(0.08-0.10)$ & $0.08(0.07-0.09)$ & - \\
\hline E.M. $\left[10^{58} \mathrm{~cm}^{-3}\right]$ & $2.5(2.2-3.0)$ & $2.1(1.4-2.8)$ & - \\
\hline$N_{\mathrm{H}}{ }^{L M C}\left[10^{21} \mathrm{~cm}^{-2}\right]$. & $4.2(3.3-5.2)$ & $1.6(0.6-2.3)$ & $3.3(2.7-4.0)$ \\
\hline Flux $_{0.5-10 \mathrm{keV}}\left[\mathrm{ergs} \mathrm{cm}^{-2} \mathrm{~s}^{-1}\right] \ldots$ & $3.2 \times 10^{-13}$ & $3.6 \times 10^{-13}$ & $6.4 \times 10^{-13}$ \\
\hline
\end{tabular}

The spectrum of Eye is hard and has no line-like structure, and is well fitted with an absorbed power-law model (Table 1). The intrinsic luminosity is $2.2 \times 10^{35} \mathrm{ergs} \mathrm{s}^{-1}$ in $0.5-10.0 \mathrm{keV}$. The central position and hard spectrum may indicate that Eye is a pulsar and/or pulsar wind nebula (PWN) of DEM L241. We searched for but could not find any coherent pulsations and time variabilities. If Eye is a pulsar and/or PWN, it belongs to the bright and hard class (Gotthelf \& Olbert 2002).

The Head and Tail emission have, on the other hand, soft and line-rich spectra. Both are well fitted with a non-equilibrium ionization collisional plasma emission model (Borkowski et al. 2001) ver. 2.0 as seen in Table 1. The over-abundant Ne and lessabundant Fe relative to the average LMC value (0.3; Russel \& Dopita 1992) indicate that the progenitor of DEM L241 is a very massive star. The estimated total plasma mass $\left(\sim 200 M_{\odot}\right)$ and the thermal energy $\left(5 \times 10^{50}\right.$ ergs $)$ also suggest the massive star origin, together with the existence of the central point source, Eye, and the OB star association, LH 88 (Chu \& Kennicutt 1988).

\section{References}

Bamba, A., Ueno, M., Nakajima, H., Mori, H., \& Koyama, K. 2005, submitted to A\&A

Borkowski, K. J., Lyerly, W. J., \& Reynolds, S. P. 2001, ApJ, 548, 820

Chu, Y. \& Kennicutt, R. C. 1988, AJ, 96, 1874

Gotthelf, E. V. \& Olbert, C. M. 2002, ASPC 271, 171

Mathewson, D. S., Ford, V. L., Tuohy, I. R., Mills, B. Y., Turtle, A. J., \& Helfand, D. J. 1985, ApJS, 58, 197

Russell, S. C. \& Dopita, M. A. 1992, ApJ, 384, 508 